RESEARCH LETTER

Volume 1 (2020), No. 3, pp.85-92

DOI: $10.15367 /$ ch.v1i3.407

\title{
An Ecologic Analysis of Comorbidities in Patients with COVID-19 in Philadelphia and New York City
}

KRYS JOHNSON, MPH, PHD ${ }^{1}$; ILENE HOLLIN, PHD ${ }^{2}$; AIMEE PALUMBO, PHD ${ }^{1}$; JACQUELINE C. SPITZER, MSED ${ }^{3}$; DAVID B. SARWER, PHD ${ }^{3,4}$

${ }^{1}$ Department of Epidemiology and Biostatistics, College of Public Health, Temple University

${ }^{2}$ Department of Health Services Administration and Policy, College of Public Health, Temple University

${ }^{3}$ Center for Obesity Research and Education, College of Public Health, Temple University

${ }^{4}$ Department of Social and Behavioral Sciences, College of Public Health, Temple University

Correspondence: dsarwer@,temple.edu (David B. Sarwer)

Objective: Early reports of the COVID-19 pandemic suggested that the coronavirus was associated with more serious disease and mortality in older adults. However, subsequent reports suggested that those with underlying comorbidities, including obesity, type 2 diabetes, heart and respiratory diseases were most at risk for more severe outcomes. As a result, most studies focused on comorbidities among those who were hospitalized or critically ill. However, there is a need to understand how common comorbidities are associated with overall risk of infection. This analysis aimed to explore the relationship between COVID-19 infection and common comorbidities. Methods: An ecologic analysis using multivariable regression explored rates of COVID-19 identification at the end of the summer of 2020 and age-adjusted, area-level estimates of health-related factors at the rip code level in both Philadelphia, PA and New York City, NY. Results: The analysis found that small area estimated prevalence of obesity and asthma were significant ecologic predictors of rates of COVID-19 cases in New York. City. In contrast, small area estimated prevalence of arthritis were significant predictors in Philadelphia. Conclusions: There are important area-level variations in COVID-19 infections that are correlated with variations in other chronic conditions, suggesting that factors that influence health disparities may be associated with the distribution of COVID-19.

\section{Introduction}

The earliest scientific reports and mass media coverage of the COVID-19 pandemic suggested that the coronavirus was associated with serious disease and mortality in older adults. ${ }^{1-3}$ For example, the casefatality rate in China was $8.0 \%$ percent in patients aged $70-79$ and $14.8 \%$ among patients 80 years or older, much higher than the overall case-fatality rate of $2.3 \% .^{2}$ As we learned more about the disease, preliminary reports suggested some middle-aged adults were experiencing higher morbidity and mortality. In Seattle, for example, the case fatality rate among critically ill persons younger than 65 years was $37 \%{ }^{4}$ Over time, it appeared that a high proportion of middle-aged adults who were hospitalized with COVID19 had significant, underlying comorbid risk factors, including obesity, type 2 diabetes, heart disease and 
respiratory disease. ${ }^{5-7}$ For example, Lighter et al. (2020) found that for people under the age of 60 , there was a 2-fold increased risk in admission for acute or intensive care for people with moderate obesity $\left(\mathrm{BMI}=30-34 \mathrm{~kg} / \mathrm{m}^{2}\right)$ compared with those with a BMI $<30 \mathrm{~kg} / \mathrm{m}^{2}$; risks were even greater among persons with BMI $>35 \mathrm{~kg} / \mathrm{m}^{2} .{ }^{8}$ Obesity was found to be an independent predictor of the need for intensive care or death across all age groups. ${ }^{8}$ Similarly, single center studies from France and Italy showed that the need for invasive mechanical ventilation (IMV) increased with BMI. ${ }^{9,10}$

The association between COVID-19 severity and pre-existing lung disease would seem intuitive, as persons with compromised lung function would be less well positioned to fend off a respiratory pathogen. The association with obesity, however, was somewhat surprising, as the health effects of obesity are more commonly seen in other organ systems. As we were learning more about the course and treatment of COVID-19, early testing data on COVID-19 served as a proxy for severity of disease. In the earliest days of the pandemic, limited testing resources were reserved for symptomatic people. As testing availability increases, and we are able to test people with less severe clinical and subclinical manifestations, it is important to understand what factors are not only associated with severe outcomes, but with mild and asymptomatic infection as well. The above mentioned studies focused on descriptions of risk factors among those who have been hospitalized or critically ill. ${ }^{1-10}$ However, there remains a need to understand how common underlying health conditions are associated with identification of COVID19.

To further explore the relationship between COVID-19 and common risk factors, including obesity, we undertook an analysis of COVID-19 cases in Philadelphia (the home city of the investigative team) and New York City as a comparator. While there were fewer cases in Philadelphia, ${ }^{11-12}$ the two urban areas share many commonalities, including racial, ethnic, and socioeconomic diversity as well as the existence of areas with high population density. ${ }^{13}$ In addition, the city and state response to the pandemic, with regard to stay-at-home orders and business closures, has been quite similar, including swift social distancing orders, increase in community testing sites, and phased reopening based on rates of new cases and hospitalizations. ${ }^{14,15}$ As the research question is exploratory, no priori hypotheses were articulated.

\section{Methods}

\section{Datasets}

Zip code-level counts of laboratory-confirmed COVID-19 cases in New York City were obtained via publicly shared GIS layers available on ArcGIS Online which included the number of cases by zip code. ${ }^{12}$ Data for Philadelphia were recorded from the Pennsylvania Department of Health dashboard ${ }^{11}$ and include all cases as of May 28, 2020, a time period which encompasses the first wave of infections in these two cities and just before initial lockdown restrictions were eased. ${ }^{16,17}$ Population estimates as well as median age, percent population of minority background, and relevant socioeconomic variables were obtained from the 2010 US Census. ${ }^{18}$ Risk factor data for specific disease and behavioral measures within the cities was obtained from the Centers for Disease Control and Prevention and Robert Wood Johnson Foundation's 500 Cities Project, which contains local data on specific health-related measures for large cities across the US. ${ }^{19}$ The 500 Cities data for 2017, the most recent year for which data were available, were likewise publicly accessible via ArcGIS and used to obtain census tract-level prevalence estimates of obesity, hypertension, diabetes, kidney disease, and other obesity-related sequelae. Poor health was defined as a self-reported estimate of the number of the last 30 days on which a person felt their physical health was poor, as collected by the CDC Healthy Days survey and reported in the 500 Cities data. In adults ages 18 and older, age-adjusted estimates were only available at the city level. To address this, 
census tract-level data were merged with median age from the 2010 Census via the GeoEnrichment Tool in ArcGIS to enable area-level age adjustment.

\section{Analysis Procedure}

Within ArcGIS, zip code-level COVID-19 files were geospatially joined with the 500 Cities census tractlevel data using the Summarize Within tool. This tool compiles the results of the 500 Cities' census tracts and mean age based on 2010 Census data within the zip code polygons to calculate zip code-level small area estimates of risk factors for chronic disease and obesity-related health outcomes including prevalence estimates of obesity, hypertension, diabetes, kidney disease, and other weight-related sequelae. Following this procedure, the data for each city were extracted to a CSV file and analyzed using SAS Version 9.4.

To standardize comparisons between zip codes, population estimates from the 2010 Census were used to calculate rates of positive COVID-19 cases per one-thousand population as the dependent variable. Variables which have been associated with COVID-19 complications (Table 1) were considered for inclusion in the ecologic analysis of each city-based multivariable regression model. Correlation analyses were conducted to assess collinearity between variables and those with strong collinearity were excluded from the multivariable regression model. Models were adjusted for median age and race (via percent population of minority background).

Table 1. Zip-code level estimates of health-related factors, Philadelphia and New York City (NYC), 2017

\begin{tabular}{|c|c|c|}
\hline & Philadelphia $(\mathrm{n}=44)$ & NYC $(n=163)$ \\
\hline & \multicolumn{2}{|c|}{ Zip-code mean or $\%(\min , \max )$} \\
\hline COVID-19 cases (per 1000 population) & $10.5(0.0,21.04)$ & $22.63(5.94,42.26)$ \\
\hline Median age (2010) & $34.88(23.6,46.2)$ & $36.53(21.1,58.5)$ \\
\hline \multicolumn{3}{|c|}{ Individual Factors and Previous History (Percent with risk factor) } \\
\hline Uninsured & $13.60(4.2,34)$ & $15.51(4.5,36.9)$ \\
\hline Current smoker & $22.98(10.9,33.4)$ & $16.08(7.2,29.3)$ \\
\hline Low physical activity & $29.23(14.6,48.7)$ & $29.32(12.8,49.9)$ \\
\hline Poor physical health & $14.58(7.7,22.1)$ & $12.17(4.9,22.5)$ \\
\hline Poor mental health & $15.76(8.9,21.4)$ & $13.06(7.6,22.4)$ \\
\hline Obesity & $32.98(21.4,46.8)$ & $24.83(11.6,44.2)$ \\
\hline \multicolumn{3}{|l|}{ Health Conditions } \\
\hline \multicolumn{3}{|l|}{ Cardiovascular } \\
\hline Hypertension & $33.9(19,50.8)$ & $27.87(9.7,47.5)$ \\
\hline Asthma & $11.23(8.6,14.8)$ & $9.69(7.1,13.8)$ \\
\hline Heart disease & $6.40(3.3,10.2)$ & $5.30 \quad(1.1,13.9)$ \\
\hline Chronic Obstructive Pulmonary Disease & $7.06(3.4,10.7)$ & $5.48(1.6,12.4)$ \\
\hline Prior stroke & $3.91(1.6,7.1)$ & $2.98(0.7,7.3)$ \\
\hline Diabetes & $12.98(5.1,21.1)$ & $10.55(2.2,21.5)$ \\
\hline Kidney disease & $3.31(1.7,5.2)$ & $2.97(1.0,5.7)$ \\
\hline Cancer & $5.80(3.6,8.1)$ & $5.43(2,11.5)$ \\
\hline
\end{tabular}




\section{Results}

Data were available from all 44 Philadelphia and 163 New York City zip codes. There was substantial variation in health-related variables by zip code in both cities, with rates of obesity ranging from $21.4 \%$ to $46.8 \%$ in Philadelphia and from $11.6 \%$ to $44.2 \%$ in New York City, as seen in Table 1. Higher prevalence of obesity was associated with increased rates of COVID-19 cases in New York City (Table 2 ); each 1\% increase in area-level prevalence of obesity was associated with just under 1 additional case of COVID-19 (per 1000 people). Conversely, prevalence of current asthma was negatively associated with COVID-19 rates in New York City. In Philadelphia, history of arthritis was the only variable that was statistically significantly associated with the rate of COVID-19 cases. The model for Philadelphia explained $24.45 \%$ of the variability in case rates, while the NYC model explained $38.63 \%$ of case rates.

Table 2. Associations between the prevalence of chronic conditions and rates of COVID-19 infections (per 1000 population), New York City (NYC) and Philadelphia, 2020. Adjusted for SES, race, and age.

\begin{tabular}{|l|c|c|c|c|c|}
\hline & Parameter & Estimate & $\begin{array}{c}\text { Standard } \\
\text { Error }\end{array}$ & Adj R Square & P-Value \\
\hline NYC & Intercept & 12.016525 & 3.427851 & 0.3863 & \\
\hline & Asthma & -1.327669 & 0.567202 & & $<.0001$ \\
\hline & Obesity & 0.945432 & 0.127227 & & 0.0205 \\
\hline Philadelphia & Intercept & -0.833805 & 29.442962 & 0.2445 & \\
\hline & Arthritis & 4.212327 & 1.142606 & & 0.0006 \\
\hline
\end{tabular}


Figure 1. Quintiles of COVID-19 cases per 100,000 for Philadelphia (top) and New York City (bottom) up to May 28,2020

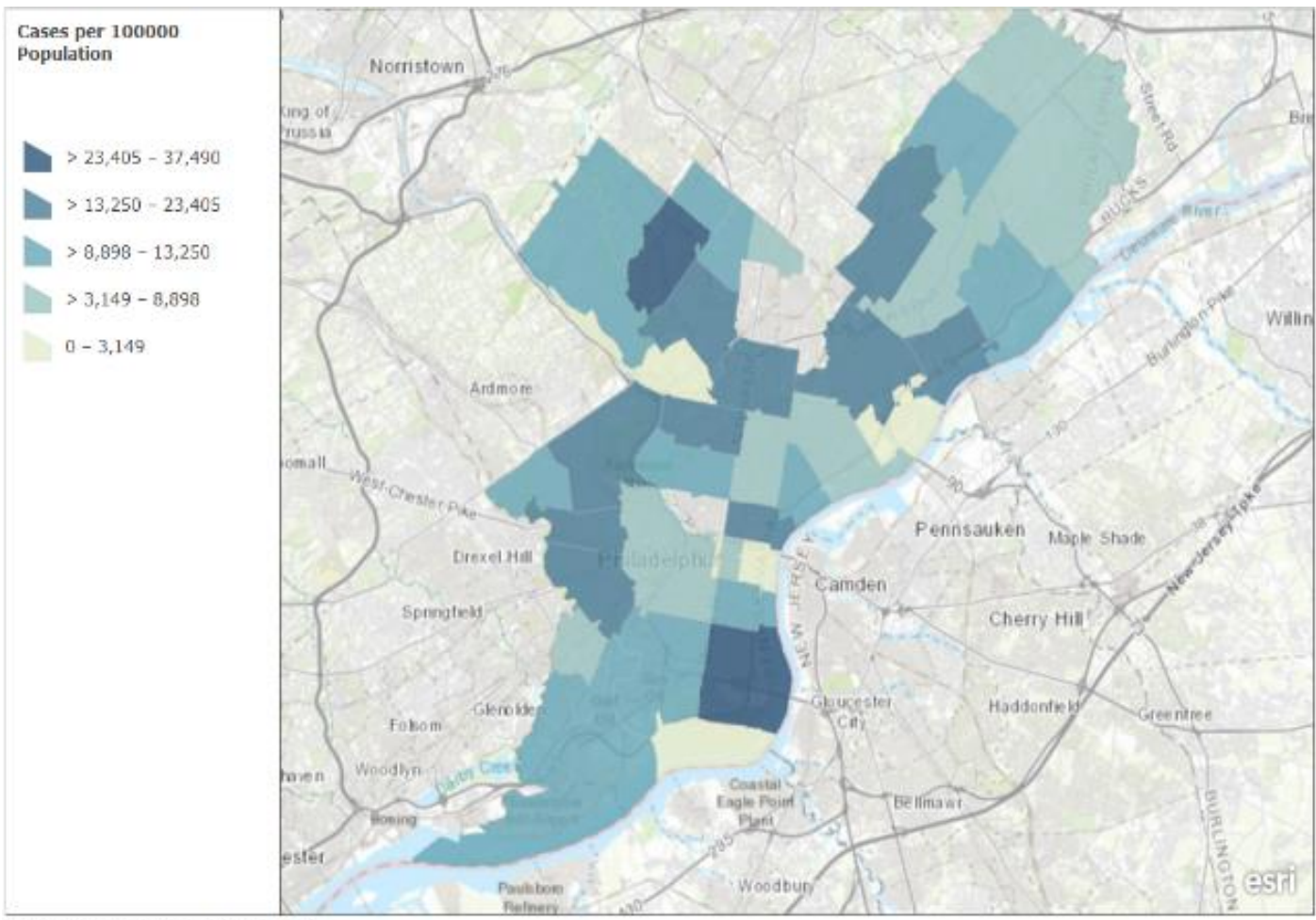

Philly and NYC covid cases by zp code

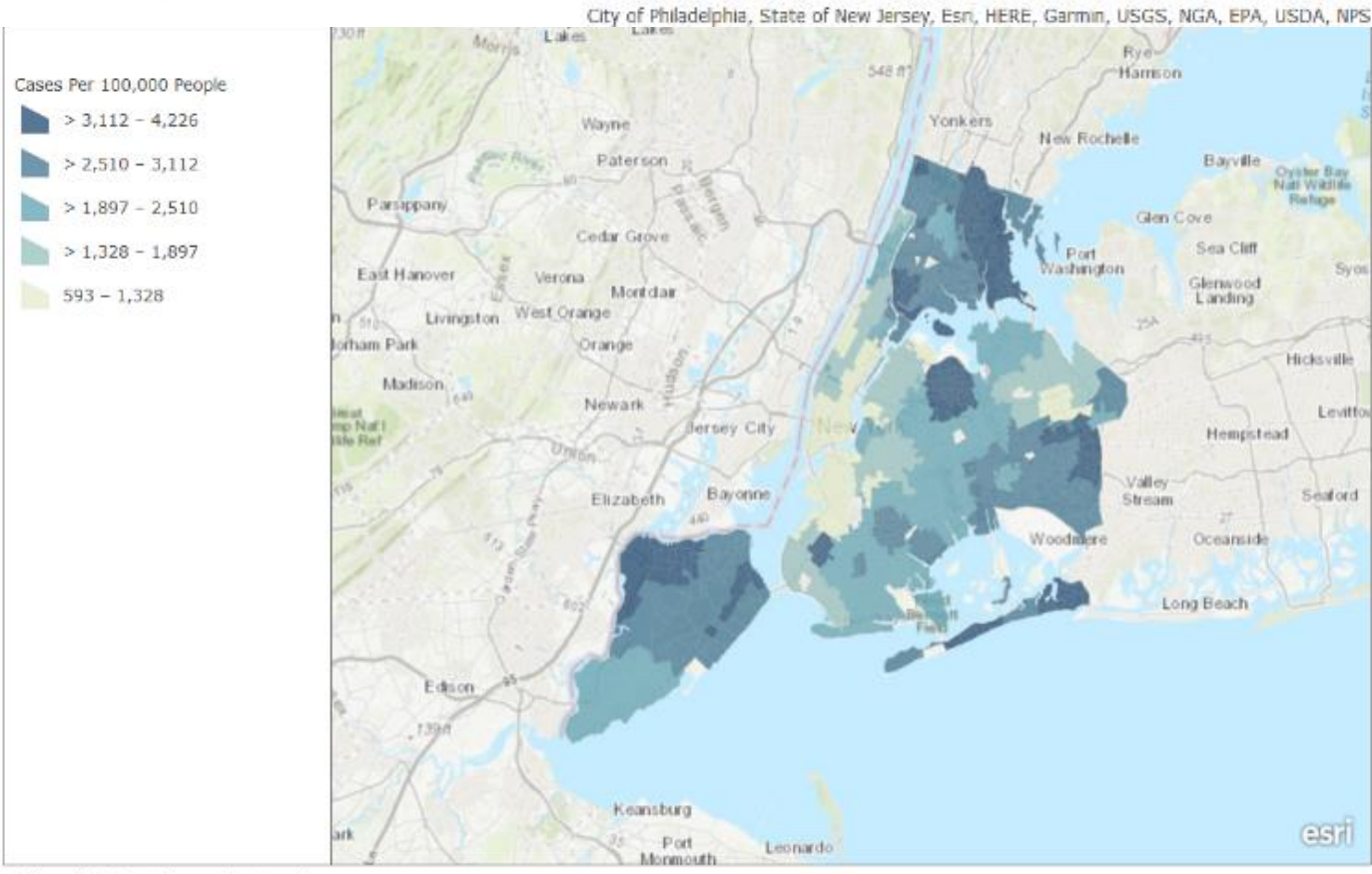

Philly and NYC covid cases by zip code

Latest maps available at: https://www.phila.gov/programs/coronavirus-disease-2019-covid-19/testingand-data/\#/ and https://www1.nyc.gov/site/doh/covid/covid-19-data.page. 


\section{Discussion}

This preliminary analysis found that small area estimated prevalence of obesity and asthma were significant, ecologic predictors of population-based rates of COVID-19 cases in New York City. In contrast, small area estimated prevalence of arthritis was a significant predictor in Philadelphia. The relationship between obesity and COVID-19 has been found in several ${ }^{5-10}$ but not all studies, ${ }^{20}$ with our investigation contributing to this growing literature.

We found an association between area-level prevalence of obesity and COVID-19 infections. However, it was small; each 1\% increase in area-level prevalence of obesity was associated with just under 1 additional case of COVID-19 in New York City (per 1000 people). As the analysis is ecologic, the conclusions should not be applied at the individual level. However, in early mass media reports of the association between COVID-19 and obesity, the relationship was individualized, with a tone that persons with obesity were at substantial risk because of their adiposity. Fortunately, a number of international thought leaders who work in the area of weight stigma and bias have been quick to note this misinterpretation and rightly categorize it as yet another, unfortunate example of how persons with obesity are blamed for their comorbidities and resulting poor physical condition. By contrast, there may be several area-level social and environmental factors that we were not able to explore that may explain the correlation we see between area-level prevalence of health-related risk factors and rates of COVID19.

There are several limitations to this analysis. This was an ecologic analysis exploring aggregate case counts across zip codes compared to area-level estimates of health-related variables and outcomes. Since these data did not include individual information on the health status of the case patients, conclusions cannot be drawn about individual risk factors. However, this analysis suggests that there are important area-level variations in COVID-19 infections that are correlated with variations in other chronic conditions, suggesting that the factors that influence health disparities may also be operating in the distribution of COVID-19.

Furthermore, there presently are limitations with overall infection rate data. The analyses were conducted with data only in the first wave of the COVID-19 pandemic in the United States; testing was limited early on and was often not available to people without a prescription. Thus, many people who had mild symptoms or who do not have a primary care physician may not have been tested and would not be counted in the data. This fact may explain the associations observed with both obesity and arthritis; rather than indicators of who is being infected, these data continue to reflect those with the most severe outcomes after infection. Arthritis is a common condition among people with other comorbidities including diabetes and heart disease, ${ }^{21}$ known risk factors for severe outcomes of COVID-19. Obesity is also a major risk factor for arthritis and the collinearity between these conditions may actually speak to the similarity of risk factors across regions rather than differences. Finally, the implementation of restrictions on travel and in-person activities may also impact overall rates of COVID-19 during this first wave; however, Philadelphia and New York City implemented similar stay-at-home orders at similar time intervals, enabling comparison of these cities during spring 2020. New and more thorough data through summer and fall 2020 may alter conclusions and clarify relationships.

In conclusion, this analysis found that small area-estimated prevalence of obesity and asthma were significant ecologic predictors of population-based rates of COVID-19 cases in New York City, but not Philadelphia, where small area -estimated prevalence of arthritis was a significant predictor of rates. Thus, area-level variations in COVID-19 infections appear to be associated with other chronic health conditions. Many of these conditions are found in higher rates in profoundly underserved neighborhoods 
of both cities, where there may be a greater density of individuals who have had to continue with inperson work during the pandemic and have fewer health care resources available to them.

\section{Disclosures and Conflicts of Interest}

Dr. Sarwer has consulting relationships with Ethicon and Novo Nordisk. He also serves as Faculty Editor of CommonHealth.

\section{Funding Sources}

Dr. Sarwer currently has grant funding from the National Institute of Diabetes, Digestive, and Kidney Disease (R01-DK-108628-01), National Institute of Dental and Craniofacial Research (R01 DE026603), Department of Defense, as well as the Commonwealth of Pennsylvania (PA CURE).

\section{References}

1. Zhou F, Yu T, Du R, et al. Clinical course and risk factors for mortality of adult inpatients with COVID-19 in Wuhan, China: a retrospective cohort study. The Lancet; 2020;395:1054-1062. doi: 10.1016/s0140-6736(20)30566-3.

2. Wu Z, McGoogan JM. Characteristics of and important lessons from the coronavirus disease 2019 (COVID-19) outbreak in China: Summary of a report of 72, 314 cases from the Chinese Center for Disease Control and Prevention. JAMA 2020;323(13):1239-42. doi: 10.1001/jama.2020.2648.

3. Grasselli G, Zangrillo A, Zanella A, et al. Baseline characteristics and outcomes of 1,591 patients infected with SARS-CoV-2 admitted to ICUs of the Lombardy Region, Italy. JAMA 2020;323(16):1574-81. doi: 10.1001/jama.2020.5394.

4. Bhatraju PK, Ghassemieh BJ, Nichols M, et al. COVID-19 in critically ill patients in the Seattle region — case series. NEJM 2020;382:2012-2022. doi: 10.1056/NEJMoa2004500.

5. Goyal P, Choi JJ, Pinheiro LC, et al. Clinical characteristics of COVID-19 in New York City. NEJM 2020. doi: 10.1056/NEJMc2010419.

6. Richardson S, Hirsch JS, Narasimhan M, Crawford JM, McGinn T, Davidson KW, et al. Presenting characteristics, comorbidities, and outcomes among 5700 patients hospitalized with COVID-19 in the New York City area. JAMA 2020;323(20):2052-2059. doi: 10.1001/jama.2020.6775.

7. Petrilli CM, Jones SA, Yang J, et al. Factors associated with hospitalization and critical illness among 4,103 patients with COVID-19 disease in New York City. medRxiv. 2020:2020.04.08.20057794. doi: 10.1101/2020.04.08.20057794.

8. Lighter J, Phillips M, Hochman S, et al. Obesity in patients younger than 60 years is a risk factor for COVID-19 hospital admission. Clin Infec Dis. 2020 71(15):896-897. doi: 10.1093/cid/ciaa415.

9. Simonnet A, Chetboun M, Poissy J, et al. High prevalence of obesity in severe acute respiratory syndrome coronavirus-2 (SARS-CoV-2) requiring invasive mechanical ventilation. Obesity 2020 in press. doi: 10.1002/oby.22831.

10. Busetto L, Bettini S, Fabris R, et al. Obesity and COVID-19: an Italian snapshot. Obesity in press. doi: 10.1002/oby.22918.11. Pennsylvania Department of Health. Pennsylvania COVID-19 Numbers. Pennsylvania Department of Health. https://www.health.pa.gov/topics/disease/coronavirus/Pages/Cases.aspx. Published 2020. Accessed May 14, 2020. 
12. NYC Health. (n.d.). COVID-19: Data Main. Retrieved May 28, 2020, from https://www1.nyc.gov/site/doh/covid/covid-19-data.page

13. United States Census Bureau QuickFacts. New York City, New York and Philadelphia County, Pennsylvania. https://www.census.gov/quickfacts/fact/table/newyorkcitynewyork,philadelphiacountypennsylva nia/PST045219. Accessed November 29, 2020.

14. McDaniel J, Orso A, and the Staff of The Philadelphia Inquirer. Months into the unknown, lightyears from normal. The Philadelphia Inquirer. 18 Sep 2020. https://www.inquirer.com/health/coronavirus/inq/coronavirus-cases-pennsylvania-philadelphianj-us-timeline-20200918.html. Accessed November 29, 2020.

15. Francescani C. Timeline: The first 100 days of New York Gov. Andrew Cuomo's COVID-19 response. 17 Jun 2020. ABCnews. https:/ /abcnews.go.com/US/News/timeline-100-days-york-govandrew-cuomos-covid/story?id=71292880. Accessed November 29, 2020.16. McDaniel J, Orso A. Months into the unknown, light-years from normal. Philadelphia Inquirer. September 18, 2020. Accessed December 3, 2020. https://www.inquirer.com/health/coronavirus/inq/coronaviruscases-pennsylvania-philadelphia-nj-us-timeline-20200918.html

17. Kerr A, A Historical Timeline of COVID-19 in New York City. Investopedia. October 6, 2020. Accessed December 3, 2020. https://www.investopedia.com/historical-timeline-of-covid-19-innew-york-city-5071986

18. US Census Bureau. (n.d.). 2010 Census Tract Data. Retrieved December 4, 2020, from https://data.census.gov/cedsci/

19. Centers for Disease Control and Prevention, National Center for Chronic Disease Prevention and Health Promotion, Division of Population Health. 500 Cities Project Data [online]. https://www.cdc.gov/500cities. Published 2018 [accessed May 14, 2020].

20. Wu J, Li W, Shi X et al. Early antiviral treatment contributes to alleviate the severity and improve the prognosis of patients with novel coronavirus disease (COVID-19). J Int Med 2020; in press. DOI: 10.1111/joim.13063

21. Centers for Disease Control and Prevention. Arthritis Comorbidities. https://www.cdc.gov/arthritis/data_statistics/comorbidities.htm. Published May 16, 2018. Accessed May 14, 2020.

\section{Statement of Contributions}

Dr. Johnson was responsible for data analysis. All authors reviewed the results and contributed to the final version of the manuscript. 